\title{
Criteria for exponential convergence to quasi-stationary distributions and applications to multi-dimensional diffusions
}

\author{
Nicolas Champagnat ${ }^{1,2,3}$, Koléhè Abdoulaye Coulibaly-Pasquier ${ }^{1,2}$, \\ Denis Villemonais ${ }^{1,2,3}$
}

August 13, 2018

\begin{abstract}
We consider general Markov processes with absorption and provide criteria ensuring the exponential convergence in total variation of the distribution of the process conditioned not to be absorbed. The first one is based on two-sided estimates on the transition kernel of the process and the second one on gradient estimates on its semigroup. We apply these criteria to multi-dimensional diffusion processes in bounded domains of $\mathbb{R}^{d}$ or in compact Riemannian manifolds with boundary, with absorption at the boundary.
\end{abstract}

Keywords: Markov processes; diffusions in Riemannian manifolds; diffusions in bounded domains; absorption at the boundary; quasi-stationary distributions; $Q$-process; uniform exponential mixing; two-sided estimates; gradient estimates.

2010 Mathematics Subject Classification. Primary: 60J60; 37A25; 60B10; 60F99. Secondary: 60J75; 60J70

\section{Introduction}

Let $X$ be a Markov process evolving in a measurable state space $E \cup\{\partial\}$ absorbed at $\partial \notin E$ at time $\tau_{\partial}=\inf \left\{t \geq 0, X_{t}=\partial\right\}$. We assume that

\footnotetext{
${ }^{1}$ IECL, Université de Lorraine, Site de Nancy, B.P. 70239, F-54506 Vanduvre-ls-Nancy Cedex, France

${ }^{2}$ CNRS, IECL, UMR 7502, Vandœuvre-lès-Nancy, F-54506, France

${ }^{3}$ Inria, TOSCA team, Villers-lès-Nancy, F-54600, France.

E-mail: Nicolas.Champagnat@inria.fr, Kolehe.Coulibaly@univ-lorraine.fr, Denis.Villemonais@univ-lorraine.fr
} 
$\mathbb{P}_{x}\left(t<\tau_{\partial}\right)>0$, for all $x \in E$ and all $t \geq 0$, where $\mathbb{P}_{x}$ is the law of $X$ with initial position $x$. We consider the problem of existence of a probability measure $\alpha$ on $E$ and of positive constants $B, \gamma>0$ such that, for all initial distribution $\mu$ on $E$,

$$
\left\|\mathbb{P}_{\mu}\left(X_{t} \in \cdot \mid t<\tau_{\partial}\right)-\alpha(\cdot)\right\|_{T V} \leq B e^{-\gamma t}, \quad \forall t \geq 0,
$$

where $\mathbb{P}_{\mu}$ is the law of $X$ with initial distribution $\mu$ and $\|\cdot\|_{T V}$ is the total variation norm on finite signed measures. It is well known that (1.1) entails that $\alpha$ is the unique quasi-stationary distribution for $X$, that is the unique probability measure satisfying

$$
\alpha(\cdot)=\mathbb{P}_{\alpha}\left(X_{t} \in \cdot \mid t<\tau_{\partial}\right), \quad \forall t \geq 0 .
$$

Our goal is to provide sufficient conditions for (1.1) with applications when $X$ is a diffusion process, absorbed at the boundary of a domain of $\mathbb{R}^{d}$ or of a Riemannian manifold. Our first result (Theorem 2.1) shows that a two-sided estimate for the transition kernel of a general absorbed Markov process is sufficient to ensure (1.1). This criterion applies in particular to diffusions with smooth coefficients in bounded domains of $\mathbb{R}^{d}$ with irregular boundary. Our second result (Theorem 3.1) concerns Markov processes satisfying gradient estimates (as in Wang [29] and Priola and Wang [26]), irreducibility conditions and controlled probability of absorption near the boundary. It applies to diffusions with less regular coefficients in smooth domains of $\mathbb{R}^{d}$ and to drifted Brownian motions in compact Riemannian manifolds with $C^{2}$ boundary.

Convergence of conditioned diffusion processes have been already obtained for diffusions in domains of $\mathbb{R}^{d}$, mainly using spectral theoretic arguments (see for instance [3, 19, 23, 24, 14, 5] for $d=1$ and $[4,18,12]$ for $d \geq 2$ ). Among these references, $[18,12]$ give the most general criteria for diffusions in dimension 2 or more. Using two-sided estimates and spectral properties of the infinitesimal generator of $X$, Knobloch and Partzsch [18] proved that (1.1) holds for a class of diffusion processes evolving in $\mathbb{R}^{d}(d \geq 3)$ with $C^{1}$ diffusion coefficient, drift in a Kato class and $C^{1,1}$ domain. In [12], the authors obtain (1.1) for diffusions with global Lipschitz coefficients (and additional local regularity near the boundary) in a domain with $C^{2}$ boundary. These results can be recovered with our method (see Section 2 and 3.2 respectively). When the diffusion is a drifted Brownian motion with drift deriving from a potential, the authors of [4] obtain existence and uniqueness results for the quasi-stationary distribution in cases with singular drifts 
and unbounded domains with non-regular boundary that do not enter the settings of this paper.

Usual tools to prove convergence in total variation for processes without absorption involve coupling arguments: for example, contraction in total variation norm for the non-conditioned semi-group can be obtained using mirror and parallel coupling, see [22, 29, 26], or lower bounds on the density of the process that could be obtained for example using Aronson-type estimates or Malliavin calculus [1, 28, 30, 25]. However, on the one hand, lower bounds on transition densities are not sufficient to control conditional distributions, and on the other hand, the process conditioned not to be killed up to a given time $t>0$ is a time-inhomogeneous diffusion process with a singular drift for which these methods fail. For instance, a standard $d$-dimensional Brownian motion $\left(B_{t}\right)_{t \geq 0}$ conditioned not to exit a smooth domain $D \subset \mathbb{R}^{d}$ up to a time $t>0$ has the law of the solution $\left(X_{s}^{(t)}\right)_{s \in[0, t]}$ to the stochastic differential equation

$$
d X_{s}^{(t)}=d B_{s}+\left[\nabla \ln \mathbb{P} .\left(t-s<\tau_{\partial}\right)\right]\left(X_{s}^{(t)}\right) d s .
$$

where the drift term is singular near the boundary. Our approach is thus to use the following condition, which is actually equivalent to the exponential convergence (1.1) (see [6, Theorem 2.1]).

Condition (A). There exist $t_{0}, c_{1}, c_{2}>0$ and a probability measure $\nu$ on $E$ such that

(A1) for all $x \in E$,

$$
\mathbb{P}_{x}\left(X_{t_{0}} \in \cdot \mid t_{0}<\tau_{\partial}\right) \geq c_{1} \nu(\cdot)
$$

(A2) for all $z \in E$ and all $t \geq 0$,

$$
\mathbb{P}_{\nu}\left(t<\tau_{\partial}\right) \geq c_{2} \mathbb{P}_{z}\left(t<\tau_{\partial}\right) .
$$

More precisely, if Condition (A) is satisfied, then, for all probability measure $\pi$ on $E$,

$$
\left\|\mathbb{P}_{\pi}\left(X_{t} \in \cdot \mid t<\tau_{\partial}\right)-\alpha(\cdot)\right\|_{T V} \leq 2\left(1-c_{1} c_{2}\right)^{\left\lfloor t / t_{0}\right\rfloor}
$$

and it implies that, for all probability measures $\pi_{1}$ and $\pi_{2}$ on $E$,

$$
\begin{aligned}
\| \mathbb{P}_{\pi_{1}}\left(X_{t} \in \cdot \mid t<\tau_{\partial}\right)-\mathbb{P}_{\pi_{2}}\left(X_{t} \in \cdot \mid\right. & \left.t<\tau_{\partial}\right) \|_{T V} \\
\leq & \frac{\left(1-c_{1} c_{2}\right)^{\left\lfloor t / t_{0}\right\rfloor}}{c\left(\pi_{1}\right) \vee c\left(\pi_{2}\right)}\left\|\pi_{1}-\pi_{2}\right\|_{T V}
\end{aligned}
$$


where $c\left(\pi_{i}\right)=\inf _{t \geq 0} \mathbb{P}_{\pi_{i}}\left(t<\tau_{\partial}\right) / \sup _{z \in E} \mathbb{P}_{z}\left(t<\tau_{\partial}\right)$ (see Appendix A for a proof of this improvement of [6, Corollary 2.2], where the same inequality is obtained with $c\left(\pi_{1}\right) \wedge c\left(\pi_{2}\right)$ instead of $\left.c\left(\pi_{1}\right) \vee c\left(\pi_{2}\right)\right)$.

Several other properties can also be deduced from Condition (A). For instance, $e^{\lambda_{0} t} \mathbb{P}_{x}\left(t<\tau_{\partial}\right)$ converges when $t \rightarrow+\infty$, uniformly in $x$, to a positive eigenfunction $\eta$ of the infinitesimal generator of $\left(X_{t}, t \geq 0\right)$ for the eigenvalue $-\lambda_{0}$ characterized by the relation $\mathbb{P}_{\alpha}\left(t<\tau_{\partial}\right)=e^{-\lambda_{0} t}, \forall t \geq 0$ [6, Proposition 2.3]. Moreover, it implies a spectral gap property [6, Corollary 2.4], the existence and exponential ergodicity of the so-called $Q$-process, defined as the process $X$ conditioned to never hit the boundary [6, Theorem 3.1] and a conditional ergodic property [7]. Note that we do not assume that $\mathbb{P}_{x}\left(\tau_{\partial}<\infty\right)=1$, which is only required in the proofs of [6] in order to obtain $\lambda_{0}>0$. Indeed, the above inequalities remain true under Condition (A), even if $\mathbb{P}_{x}\left(\tau_{\partial}<+\infty\right)<1$ for some $x \in E$. The only difference is that, in this case, $E^{\prime}:=\left\{x \in E, \mathbb{P}_{x}\left(\tau_{\partial}<+\infty\right)=0\right\}$ is non-empty, $\alpha$ is a classical stationary distribution such that $\alpha\left(E^{\prime}\right)=1$ and $\lambda_{0}=0$.

The paper is organized as follows. In Section 2, we state and prove a sufficient criterion for (1.1) based on two-sided estimates. In Section 3.1, we prove (1.1) for Markov processes satisfying gradient estimates, irreducibility conditions and controled probability of absorption near the boundary. In Section 3.2, we apply this result to diffusions in smooth domains of $\mathbb{R}^{d}$ and to drifted Brownian motions in compact Riemannian manifolds with smooth boundary. Section 3.3 is devoted to the proof of the criterion of Section 3.1. Finally, Appendix A gives the proof of (1.2).

\section{Quasi-stationary behavior under two-sided esti- mates}

In this section, we consider as in the introduction a general absorbed Markov process $X$ in $E \cup\{\partial\}$ satisfying two-sided estimates: there exist a time $t_{0}>0$, a constant $c>0$, a positive measure $\mu$ on $E$ and a measurable function $f: E \rightarrow(0,+\infty)$ such that

$$
c^{-1} f(x) \mu(\cdot) \leq \mathbb{P}_{x}\left(X_{t_{0}} \in \cdot\right) \leq c f(x) \mu(\cdot), \forall x \in E .
$$

Note that this implies that $f(x) \mu(E) \leq c$ for all $x \in E$, hence $\mu$ is finite and $f$ is bounded. As a consequence, one can assume without loss of generality that $\mu$ is a probability measure and then $\|f\|_{\infty} \leq c$. Note also that $f(x)>0$ for all $x \in E$ entails that $\mathbb{P}_{x}\left(t_{0}<\tau_{\partial}\right)>0$ for all $x \in E$ and hence, by 
Markov property, that $\mathbb{P}_{x}\left(t<\tau_{\partial}\right)>0$ for all $x \in E$ and all $t>0$, as needed to deduce (1.1) from Condition (A) (see [6]).

Estimates of the form (2.1) are well known for diffusion processes in a bounded domain of $\mathbb{R}^{d}$ since the seminal paper of Davies and Simon [11]. The case of standard Brownian motion in a bounded $C^{1,1}$ domain of $\mathbb{R}^{d}, d \geq 3$ was studied in [31]. This result has then been extended in [17] to diffusions in a bounded $C^{1,1}$ domain in $\mathbb{R}^{d}, d \geq 3$, with infinitesimal generator

$$
L=\frac{1}{2} \sum_{i, j=1}^{d} a_{i j} \partial_{i} \partial_{j}+\sum_{i=1}^{d} b_{i} \partial_{i},
$$

with symmetric, uniformly elliptic and $C^{1}$ diffusion matrix $\left(a_{i j}\right)_{1 \leq i, j \leq d}$, and with drift $\left(b_{i}\right)_{1 \leq i \leq d}$ in the Kato class $K_{d, 1}$, which contains $L^{p}(d x)$ functions for $p>d$. Diffusions on bounded, closed Riemannian manifolds with irregular boundary and with generator

$$
L=\Delta+X
$$

where $\Delta$ is the Laplace-Beltrami operator and $X$ is a smooth vector field, were also studied in [21]. Two-sided estimates are also known for processes with jumps $[9,2,8,16,10]$.

Theorem 2.1. Assume that there exist a time $t_{0}>0$, a constant $c>0$, a probability measure $\mu$ on $E$ and a measurable function $f: E \rightarrow(0,+\infty)$ such that (2.1) holds. Then Condition (A) is satisfied with $\nu=\mu, c_{1}=c^{-2}$ and $c_{2}=c^{-3} \mu(f)$. In addition, for all probability measures $\pi_{1}$ and $\pi_{2}$ on $E$, we have

$$
\begin{aligned}
\| \mathbb{P}_{\pi_{1}}\left(X_{t} \in \cdot \mid t<\tau_{\partial}\right)-\mathbb{P}_{\pi_{2}}\left(X_{t}\right. & \left.\in \cdot \mid t<\tau_{\partial}\right) \|_{T V} \\
& \leq c^{3} \frac{\left(1-c^{-5} \mu(f)\right)^{\left\lfloor t / t_{0}\right\rfloor}}{\pi_{1}(f) \vee \pi_{2}(f)}\left\|\pi_{1}-\pi_{2}\right\|_{T V},
\end{aligned}
$$

Moreover, the unique quasi-stationary distribution $\alpha$ for $X$ satisfies

$$
c^{-2} \mu \leq \alpha \leq c^{2} \mu .
$$

Remark 1. Recall that to any quasi-stationary distribution $\alpha$ is associated an eigenvalue $-\lambda_{0} \leq 0$. We deduce from the two-sided estimate (2.1) and [6, Corollary 2.4] an explicit estimate on the second spectral gap of the infinitesimal generator $L$ of $X$ (defined as acting on bounded measurable functions on $E \cup\{\partial\}$ ): for all $\lambda$ in the spectrum of $L$ such that $\lambda \notin\left\{0, \lambda_{0}\right\}$, the real part of $\lambda$ is smaller than $-\lambda_{0}+t_{0}^{-1} \log \left(1-c^{-5} \mu(f)\right)$. 
Remark 2. In particular, we recover the results of Knobloch and Partzsch [18]. They proved that (1.1) holds for a class of diffusion processes evolving in $\mathbb{R}^{d}$ $(d \geq 3)$, assuming continuity of the transition density, existence of ground states and the existence of a two-sided estimate involving the ground states of the generator. Similar results were obtained in the one-dimensional case in [24].

Proof of Theorem 2.1. We deduce from (2.1) that, for all $x \in E$,

$$
c^{-2} \mu(\cdot) \leq \mathbb{P}_{x}\left(X_{t_{0}} \in \cdot \mid t_{0}<\tau_{\partial}\right)=\frac{\mathbb{P}_{x}\left(X_{t_{0}} \in \cdot\right)}{\mathbb{P}_{x}\left(X_{t_{0}} \in E\right)} \leq c^{2} \mu(\cdot) .
$$

We thus obtain (A1) with $c_{1}=c^{-2}$ and $\nu=\mu$.

Moreover, for any probability measure $\pi$ on $E$ and any $z \in E$,

$$
\begin{aligned}
\mathbb{P}_{\pi}\left(X_{t_{0}} \in \cdot\right) & \geq c^{-1} \pi(f) \mu(\cdot) \\
& \geq \frac{f(z)}{\|f\|_{\infty}} c^{-1} \pi(f) \mu(\cdot) \\
& \geq c^{-3} \pi(f) \mathbb{P}_{z}\left(X_{t_{0}} \in \cdot\right) .
\end{aligned}
$$

Hence, for all $t \geq t_{0}$, we have by Markov's property

$$
\begin{aligned}
\mathbb{P}_{\pi}\left(t<\tau_{\partial}\right) & =\mathbb{E}_{\pi}\left(\mathbb{P}_{X_{t_{0}}}\left(t-t_{0}<\tau_{\partial}\right)\right) \\
& \geq c^{-3} \pi(f) \mathbb{E}_{z}\left(\mathbb{P}_{X_{t_{0}}}\left(t-t_{0}<\tau_{\partial}\right)\right) \\
& =c^{-3} \pi(f) \mathbb{P}_{z}\left(t<\tau_{\partial}\right)
\end{aligned}
$$

When $t \leq t_{0}$, we have $\mathbb{P}_{\pi}\left(t<\tau_{\partial}\right) \geq \mathbb{P}_{\pi}\left(t_{0}<\tau_{\partial}\right) \geq c^{-1} \pi(f) \geq c^{-3} \pi(f)$ and hence $\mathbb{P}_{\pi}\left(t<\tau_{\partial}\right) \geq c^{-3} \pi(f) \mathbb{P}_{z}\left(t<\tau_{\partial}\right)$, so that

$$
c(\pi):=\inf _{t \geq 0} \frac{\mathbb{P}_{\pi}\left(t<\tau_{\partial}\right)}{\sup _{z \in E} \mathbb{P}_{z}\left(t<\tau_{\partial}\right)} \geq c^{-3} \pi(f) .
$$

Taking $\pi=\nu=\mu$, this entails (A2) for $c_{2}=c^{-3} \mu(f)$ and (1.2) implies (2.2). The inequality (2.3) then follows from (2.4).

\section{Quasi-stationary behavior under gradient esti- mates}

In this section, we explain how gradient estimates on the semi-group of the Markov process $\left(X_{t}, t \geq 0\right)$ imply the exponential convergence (1.1). 


\subsection{A general result}

We assume that the process $X$ is a strong Markov, continuous ${ }^{1}$ process and we assume that its state space $E \cup\{\partial\}$ is a compact metric space with metric $\rho$ equipped with its Borel $\sigma$-field. Recall that $\partial$ is absorbing and that we assume that $\mathbb{P}_{x}\left(t<\tau_{\partial}\right)>0$ for all $x \in E$ and $t \geq 0$. Our result holds true under three conditions: first, we assume that there exists $t_{1}>0$ such that the process satisfies a gradient estimate of the form: for all bounded measurable function $f: E \cup\{\partial\} \rightarrow \mathbb{R}$

$$
\left\|\nabla P_{t_{1}} f\right\|_{\infty} \leq C\|f\|_{\infty},
$$

where $P_{t} f(x)=\mathbb{E}_{x}\left(f\left(X_{t}\right) \mathbb{1}_{t<\tau_{\partial}}\right)$ denotes the Dirichlet semi-group of $X$ and the (a bit informal in such a general setting) notation $\left\|\nabla P_{t_{1}} f\right\|_{\infty}$ has to be understood as

$$
\left\|\nabla P_{t_{1}} f\right\|_{\infty}:=\sup _{x, y \in E \cup\{\partial\}} \frac{\left|P_{t_{1}} f(x)-P_{t_{1}} f(y)\right|}{\rho(x, y)} .
$$

Second, we assume that there exist a compact subset $K$ of $E$ and a constant $C^{\prime}>0$ such that, for all $x \in E$,

$$
\mathbb{P}_{x}\left(T_{K} \leq t_{1}<\tau_{\partial}\right) \geq C^{\prime} \rho_{\partial}(x),
$$

where $\rho_{\partial}(x):=\rho(x, \partial)$ and $T_{K}=\inf \left\{t \geq 0, X_{t} \in K\right\}$. Finally, we need the following irreducibility condition: for all $x, y \in E$ and all $r>0$,

$$
\mathbb{P}_{x}\left(X_{s} \in B(y, r), \forall s \in\left[t_{1}, 2 t_{1}\right]\right)>0,
$$

where $B(y, r)$ denotes the ball of radius $r$ centered at $y$.

Theorem 3.1. Assume that the process $\left(X_{t}, t \geq 0\right)$ satisfies (3.1), (3.2) and (3.3) for some constant $t_{1}>0$. Then Condition (A) and hence (1.1) are satisfied. Moreover, there exist two constants $B, \gamma>0$ such that, for any initial distributions $\mu_{1}$ and $\mu_{2}$ on $E$,

$$
\begin{aligned}
\| \mathbb{P}_{\mu_{1}}\left(X_{t} \in \cdot \mid t<\tau_{\partial}\right)-\mathbb{P}_{\mu_{2}}\left(X_{t} \in \cdot \mid\right. & \left.t<\tau_{\partial}\right) \|_{T V} \\
\leq & \frac{B e^{-\gamma t}}{\mu_{1}\left(\rho_{\partial}\right) \vee \mu_{2}\left(\rho_{\partial}\right)}\left\|\mu_{1}-\mu_{2}\right\|_{T V} .
\end{aligned}
$$

The proof of this result is given in Section 3.3.

\footnotetext{
${ }^{1}$ The assumption of continuity is only used to ensure that the entrance times in compact sets are stopping times for the natural filtration (cf. e.g. [20, p. 48]), and hence that the strong Markov property applies at this time. Our result would also hold true for càdlàg (weak) Markov processes provided that the strong Markov property applies at the hitting times of compact sets.
} 


\subsection{The case of diffusions in compact Riemannian manifolds}

In this section, we provide two sets of assumptions for diffusions in compact manifolds with boundary $M$ absorbed at the boundary $\partial M$ (i.e. $E=M \backslash \partial M$ and $\partial=\{\partial M\})$ to which the last theorem applies:

S1. $M$ is a bounded, connected and closed $C^{2}$ Riemannian manifold with $C^{2}$ boundary $\partial M$ and the infinitesimal generator of the diffusion process $X$ is given by $L=\frac{1}{2} \Delta+Z$, where $\Delta$ is the Laplace-Beltrami operator and $Z$ is a $C^{1}$ vector field.

S2. $M$ is a compact subset of $\mathbb{R}^{d}$ with non-empty, connected interior and $C^{2}$ boundary $\partial M$ and $X$ is solution to the SDE $d X_{t}=s\left(X_{t}\right) d B_{t}+$ $b\left(X_{t}\right) d t$, where $\left(B_{t}, t \geq 0\right)$ is a $r$-dimensional Brownian motion, $b$ : $M \rightarrow \mathbb{R}^{d}$ is bounded and continuous and $s: M \rightarrow \mathbb{R}^{d \times r}$ is continuous, $s s^{*}$ is uniformly elliptic and for all $r>0$,

$$
\sup _{x, y \in M,|x-y|=r} \frac{|s(x)-s(y)|^{2}}{r} \leq g(r)
$$

for some function $g$ such that $\int_{0}^{1} g(r) d r<\infty$.

Note that (3.5) is satisfied as soon as $s$ is uniformly $\alpha$-Hölder on $M$ for some $\alpha>0$.

Let us now check that Theorem 3.1 applies in both situations.

First, the gradient estimate (3.1) is satisfied (see Wang in [29] and Priola and Wang in [26], respectively). These two references actually give a stronger version of (3.1):

$$
\left\|\nabla P_{t} f\right\|_{\infty} \leq \frac{c}{1 \wedge \sqrt{t}}\|f\|_{\infty}, \quad \forall t>0
$$

The set of assumptions S2 is not exactly the same as in [26], but they clearly imply (i), (ii), (iv) of [26, Hyp. 4.1] (see [26, Lemma 3.3] for the assumption on $s$ ) and, since we assume that $M$ is bounded and $C^{2}$, assumptions (iii') and (v) are also satisfied (see [26, Rk. 4.2]). Moreover, the gradient estimate of [26] is stated for $x \in M \backslash \partial M \mapsto P_{t} f(x)$, but can be easily extended to $x \in M$ since $P_{t} f(x) \rightarrow 0$ when $x \rightarrow \partial M$. Note also that in both references, the gradient estimates are obtained for not necessarily compact manifolds.

The irreducibility assumption (3.3) is an immediate consequence of classical support theorems [27, Exercise 6.7.5] for any value of $t_{1}>0$.

It only remains to prove the next lemma. 
Lemma 3.2. There exist $t_{1}, \varepsilon, C^{\prime}>0$ such that, for all $x \in M$,

$$
\mathbb{P}_{x}\left(T_{\varepsilon} \leq t_{1}<\tau_{\partial}\right) \geq C^{\prime} \rho_{\partial M}(x),
$$

where $\rho_{\partial M}(x)$ is the distance between $x$ and $\partial M, T_{\varepsilon}=\inf \left\{t \geq 0, X_{t} \in M_{\varepsilon}\right\}$ and the compact set $M_{\varepsilon}$ is defined as $\left\{x \in M: \rho_{\partial M}(x) \geq \varepsilon\right\}$.

Proof of Lemma 3.2. Let $\varepsilon_{0}>0$ be small enough for $\rho_{\partial M}$ to be $C^{2}$ on $M \backslash M_{\varepsilon_{0}}$. For all $t<T_{\varepsilon_{0}}$, we define $Y_{t}=\rho_{\partial M}\left(X_{t}\right)$. In both situations S1 and $\mathrm{S} 2$, we have

$$
d Y_{t}=\sigma_{t} d W_{t}+b_{t} d t
$$

where $W$ is a standard Brownian motion, where $\sigma_{t} \in[\underline{\sigma}, \bar{\sigma}]$ and $\left|b_{t}\right| \leq \bar{b}$ are adapted continuous processes, with $0<\underline{\sigma}, \bar{\sigma}, \bar{b}<\infty$. There exists a differentiable time-change $\tau(s)$ such that $\tau(0)=0$ and

$$
\widetilde{W}_{s}:=\int_{0}^{\tau(s)} \sigma_{t} d W_{t}
$$

is a Brownian motion and $\tau^{\prime}(s) \in\left[\bar{\sigma}^{-2}, \underline{\sigma}^{-2}\right]$. In addition,

$$
\int_{0}^{\tau(s)} b_{t} d t \geq-\bar{b} \tau(s) \geq-\bar{b} \underline{\sigma}^{-2} s .
$$

As a consequence, setting $Z_{s}=Y_{0}+\widetilde{W}_{s}-\bar{b} \underline{\sigma}^{-2} s$, we have almost surely $Z_{s} \leq Y_{\tau(s)}$ for all $s$ such that $\tau(s) \leq T_{\varepsilon_{0}}$.

Setting $a=\bar{b} \underline{\sigma}^{-2}$, the function

$$
f(x)=\frac{e^{2 a x}-1}{2 a}
$$

is a scale function for the drifted Brownian motion $Z$. The diffusion process defined by $N_{t}=f\left(Z_{t}\right)$ is a martingale and its speed measure is given by $s(d v)=\frac{d v}{(1+2 a v)^{2}}$. The Green formula for one-dimensional diffusion processes [15, Lemma 23.10] entails, for $\varepsilon_{1}=f\left(\varepsilon_{0}\right)$ and all $u \in\left(0, \varepsilon_{1} / 2\right)$ (in the following lines, $\mathbb{P}_{u}^{N}$ denotes the probability with respect to $N$ with initial position $N_{0}=u$ ),

$$
\begin{aligned}
\mathbb{P}_{u}^{N}\left(t \leq T_{0}^{N} \wedge T_{\varepsilon_{1} / 2}^{N}\right) & \leq \frac{\mathbb{E}_{u}^{N}\left(T_{0}^{N} \wedge T_{\varepsilon_{1} / 2}^{N}\right)}{t}=\frac{2}{t} \int_{0}^{\varepsilon_{1} / 2}\left(1-\frac{u \vee v}{\varepsilon_{1} / 2}\right)(u \wedge v) s(d v) \\
& \leq u \frac{C_{\varepsilon_{1}}}{t}, \text { where } C_{\varepsilon_{1}}=2 \int_{0}^{\varepsilon_{1} / 2} \frac{d v}{(1+2 a v)^{2}}
\end{aligned}
$$


where we set $T_{\varepsilon}^{N}=\inf \left\{t \geq 0, N_{t}=\varepsilon\right\}$. Let us fix $s_{1}=\varepsilon_{1} C_{\varepsilon_{1}}$. Since $N$ is a martingale, we have, for all $u \in\left(0, \varepsilon_{1} / 2\right)$,

$$
\begin{aligned}
u=\mathbb{E}_{u}^{N}\left(N_{\left.s_{1} \wedge T_{\varepsilon_{1} / 2}^{N} \wedge T_{0}^{N}\right)}\right. & \leq \frac{\varepsilon_{1}}{2} \mathbb{P}_{u}^{N}\left(T_{\varepsilon_{1} / 2}^{N} \leq s_{1} \wedge T_{0}^{N}\right)+\frac{\varepsilon_{1}}{2} \mathbb{P}_{u}^{N}\left(s_{1}<T_{\varepsilon_{1} / 2}^{N} \wedge T_{0}^{N}\right) \\
& \leq \frac{\varepsilon_{1}}{2} \mathbb{P}_{u}^{N}\left(T_{\varepsilon_{1} / 2}^{N} \leq s_{1} \wedge T_{0}^{N}\right)+\frac{u}{2}
\end{aligned}
$$

Hence there exists a constant $A>0$ such that $\mathbb{P}_{u}^{N}\left(T_{\varepsilon_{1} / 2}^{N} \leq s_{1} \wedge T_{0}^{N}\right) \geq A u$, or, in other words,

$$
\mathbb{P}_{x}\left(T_{\varepsilon}^{Z} \leq \underline{\sigma}^{2} t_{1} \wedge T_{0}^{Z}\right) \geq A f\left(\rho_{\partial M}(x)\right) \geq A \rho_{\partial M}(x)
$$

for all $x \in M \backslash M_{\varepsilon}$, where $t_{1}=s_{1} \underline{\sigma}^{-2}$ and $\varepsilon=f^{-1}\left(\varepsilon_{1} / 2\right)$.

Now, using the fact that the derivative of the time change $\tau(s)$ belongs to $\left[\bar{\sigma}^{-2}, \underline{\sigma}^{-2}\right]$ and that $Z_{s} \leq Y_{\tau(s)}$, it follows that for all $x \in M \backslash M_{\varepsilon}$,

$$
\mathbb{P}_{x}\left(T_{\varepsilon}^{Y} \leq t_{1} \wedge T_{0}^{Y}\right) \geq \mathbb{P}_{x}\left(T_{\varepsilon}^{Z} \leq \underline{\sigma}^{2} t_{1} \wedge T_{0}^{Z}\right) \geq A \rho_{\partial M}(x) .
$$

Therefore,

$$
\begin{aligned}
\mathbb{P}_{x}\left(T_{\varepsilon}^{Y} \leq t_{1}<T_{0}^{Y}\right) & \geq \mathbb{E}_{x}\left[\mathbb{1}_{T_{\varepsilon}^{Y} \leq t_{1} \wedge T_{0}^{Y}} \mathbb{P}_{X_{T_{\varepsilon}^{Y}}}\left(t_{1}<\tau_{\partial}\right)\right] \\
& \geq \mathbb{P}_{x}\left(T_{\varepsilon}^{Y} \leq t_{1} \wedge T_{0}^{Y}\right) \inf _{y \in M_{\varepsilon}} \mathbb{P}_{y}\left(t_{1}<\tau_{\partial}\right) \geq C^{\prime} \rho_{\partial M}(x),
\end{aligned}
$$

where we used that $\inf _{y \in M_{\varepsilon}} \mathbb{P}_{y}\left(t_{1}<\tau_{\partial}\right)>0$. This last fact follows from the inequality $\mathbb{P}_{y}\left(t_{1}<\tau_{\partial}\right)>0$ for all $y \in M \backslash \partial M$, consequence of (3.3) and from the Lipschitz-continuity of $y \mapsto \mathbb{P}_{y}\left(t_{1}<\tau_{\partial}\right)=P_{t_{1}} \mathbb{1}_{E}(y)$, consequence of (3.6).

Finally, since $T_{\varepsilon}=0$ under $\mathbb{P}_{x}$ for all $x \in M_{\varepsilon}$, replacing $C^{\prime}$ by $C^{\prime} \wedge$ $\left[\inf _{y \in M_{\varepsilon}} \mathbb{P}_{y}\left(t_{1}<\tau_{\partial}\right) / \operatorname{diam}(M)\right]$ entails (3.7) for all $x \in M$.

Remark 3. The gradient estimates of [26] are proved for diffusion processes with space-dependent killing rate $V: M \rightarrow[0, \infty)$. More precisely, they consider infinitesimal generators of the form

$$
L=\frac{1}{2} \sum_{i, j=1}^{d}\left[s s^{*}\right]_{i j} \partial_{i} \partial_{j}+\sum_{i=1}^{d} b_{i} \partial_{i}-V
$$

with $V$ bounded measurable. Our proof also applies to this setting. 
Remark 4. We have proved in particular that Condition (A1) is satisfied in situations S1 and S2. This is a minoration of conditional distributions of the diffusion. For initial positions in compact subsets of $M \backslash \partial M$, this reduces to a lower bound for the (unconditioned) distribution of the process. Such a result could be obtained from density lower bounds using number of techniques, for example Aronson-type estimates [1, 28, 30] or continuity properties [13]. Note that our result does not rely on such techniques, since it will appear in the proof that Conditions (3.1) and (3.3) are sufficient to obtain $\mathbb{P}_{x}\left(X_{t_{0}} \in \cdot\right) \geq \widetilde{\nu}$ for all $x \in M_{\varepsilon}$ for some positive measure $\widetilde{\nu}$.

\subsection{Proof of Theorem 3.1}

The proof is based on the following equivalent form of Condition (A) (see [6, Thm. 2.1])

Condition (A'). There exist $t_{0}, c_{1}, c_{2}>0$ such that

(A1') for all $x, y \in E$, there exists a probability measure $\nu_{x, y}$ on $E$ such that

$$
\mathbb{P}_{x}\left(X_{t_{0}} \in \cdot \mid t_{0}<\tau_{\partial}\right) \geq c_{1} \nu_{x, y}(\cdot) \quad \text { and } \quad \mathbb{P}_{y}\left(X_{t_{0}} \in \cdot \mid t_{0}<\tau_{\partial}\right) \geq c_{1} \nu_{x, y}(\cdot)
$$

(A2') for all $x, y, z \in E$ and all $t \geq 0$,

$$
\mathbb{P}_{\nu_{x, y}}\left(t<\tau_{\partial}\right) \geq c_{2} \mathbb{P}_{z}\left(t<\tau_{\partial}\right)
$$

Note that (A1') is a kind of coupling for conditional laws of the Markov process starting from different initial conditions. It is thus natural to use gradient estimates to prove such conditions since they are usually obtained by coupling of the paths of the process (see [29, 26]).

We divide the proof into four steps. In the first one, we obtain a lower bound for $\mathbb{P}_{x}\left(X_{2 t_{1}} \in K \mid 2 t_{1}<\tau_{\partial}\right)$. The second and third ones are devoted to the proof of $\left(\mathrm{A}^{\prime}\right)$ and $\left(\mathrm{A} 2^{\prime}\right)$, respectively. The last one gives the proof of (3.4).

\subsubsection{Return to a compact conditionally on non-absorption}

The gradient estimate (3.1) applied to $f=\mathbb{1}_{E}$ implies that $P_{t_{1}} \mathbb{1}_{E}$ is Lipschitz. Since $\mathbb{P}_{\partial}\left(t_{1}<\tau_{\partial}\right)=0$, we obtain, for all $x \in E$,

$$
\mathbb{P}_{x}\left(t_{1}<\tau_{\partial}\right) \leq C \rho_{\partial}(x) .
$$


Combining this with Assumption (3.2), we deduce that, for all $x \in E$,

$$
\mathbb{P}_{x}\left(T_{K} \leq t_{1} \mid t_{1}<\tau_{\partial}\right)=\frac{\mathbb{P}_{x}\left(T_{K} \leq t_{1}<\tau_{\partial}\right)}{\mathbb{P}_{x}\left(t_{1}<\tau_{\partial}\right)} \geq \frac{C^{\prime}}{C}
$$

Fix $x_{0} \in K$ and let $r_{0}=d\left(x_{0}, \partial\right) / 2$. We can assume without loss of generality that $B\left(x_{0}, r_{0}\right) \subset K$, since Assumption (3.2) remains true if one replaces $K$ by the set $K \cup \overline{B\left(x_{0}, r_{0}\right)} \subset E$ (which is also a compact set, as a closed subset of the compact set $E \cup\{\partial\}$ ). Then, it follows from (3.3) that, for all $x \in E$,

$\mathbb{E}_{x}\left[\mathbb{P}_{X_{t_{1}}}\left(X_{s} \in B\left(x_{0}, r_{0}\right), \forall s \in\left[0, t_{1}\right]\right)\right]=\mathbb{P}_{x}\left(X_{s} \in B\left(x_{0}, r_{0}\right), \forall s \in\left[t_{1}, 2 t_{1}\right]\right)>0$.

Because of (3.1), the left-hand side is continuous w.r.t. $x \in M$, and hence $\inf _{x \in K} \mathbb{P}_{x}\left(X_{s} \in K, \forall s \in\left[t_{1}, 2 t_{1}\right]\right) \geq \inf _{x \in K} \mathbb{P}_{x}\left(X_{s} \in B\left(x_{0}, r_{0}\right), \forall s \in\left[t_{1}, 2 t_{1}\right]\right)>0$.

Therefore, it follows from the strong Markov property at time $T_{K}$ that

$$
\begin{aligned}
\mathbb{P}_{x}\left(X_{2 t_{1}} \in K \mid 2 t_{1}<\tau_{\partial}\right) & \geq \frac{\mathbb{P}_{x}\left(X_{2 t_{1}} \in K\right)}{\mathbb{P}_{x}\left(t_{1}<\tau_{\partial}\right)} \\
& \geq \frac{\mathbb{P}_{x}\left(T_{K} \leq t_{1} \text { and } X_{T_{K}+s} \in K, \forall s \in\left[t_{1}, 2 t_{1}\right]\right)}{\mathbb{P}_{x}\left(t_{1}<\tau_{\partial}\right)} \\
& \geq \inf _{x \in K} \mathbb{P}_{x}\left(X_{s} \in K, \forall s \in\left[t_{1}, 2 t_{1}\right]\right) \frac{\mathbb{P}_{x}\left(T_{K} \leq t_{1}\right)}{\mathbb{P}_{x}\left(t_{1}<\tau_{\partial}\right)}
\end{aligned}
$$

Therefore, we have proved that, for all $x \in E$,

$$
\mathbb{P}_{x}\left(X_{2 t_{1}} \in K \mid 2 t_{1}<\tau_{\partial}\right) \geq A,
$$

for the positive constant $A:=\inf _{x \in K} \mathbb{P}_{x}\left(X_{s} \in K, \forall s \in\left[t_{1}, 2 t_{1}\right]\right) C^{\prime} / C$.

\subsubsection{Proof of (A1')}

For all $x, y \in E$, let $\mu_{x, y}$ be the infimum measure of $\delta_{x} P_{2 t_{1}}$ and $\delta_{y} P_{2 t_{1}}$, i.e. for all measurable $A \subset E$,

$$
\mu_{x, y}(A):=\inf _{A_{1} \cup A_{2}=A, A_{1}, A_{2} \text { measurable }}\left(\delta_{x} P_{2 t_{1}} \mathbb{1}_{A_{1}}+\delta_{y} P_{2 t_{1}} \mathbb{1}_{A_{2}}\right) .
$$

The proof of (A1') is based on the following lemma.

Lemma 3.3. For all bounded continuous function $f: E \rightarrow \mathbb{R}_{+}$not identically 0 , the function $(x, y) \in E^{2} \mapsto \mu_{x, y}(f)$ is Lipschitz and positive. 
Proof. By (3.1), for all bounded measurable $g: E \rightarrow \mathbb{R}$,

$$
\left\|\nabla P_{2 t_{1}} g\right\|_{\infty}=\left\|\nabla P_{t_{1}}\left(P_{t_{1}} g\right)\right\|_{\infty} \leq C\left\|P_{t_{1}} g\right\|_{\infty} \leq C\|g\|_{\infty} .
$$

Hence, for all $x, y \in E$,

$$
\left|P_{2 t_{1}} g(x)-P_{2 t_{1}} g(y)\right| \leq C\|g\|_{\infty} \rho(x, y) .
$$

This implies the uniform Lipschitz-continuity of $P_{2 t_{1}} g$. In particular, we deduce that

$$
\mu_{x, y}(f)=\inf _{A_{1} \cup A_{2}=E}\left\{P_{2 t_{1}}\left(f \mathbb{1}_{A_{1}}\right)(x)+P_{2 t_{1}}\left(f \mathbb{1}_{A_{2}}\right)(y)\right\}
$$

is continuous w.r.t. $(x, y) \in E^{2}$ (and even Lipschitz).

Let us now prove that $\mu_{x, y}(f)>0$. Let us define $\bar{\mu}_{x, y}$ as the infimum measure of $\delta_{x} P_{t_{1}}$ and $\delta_{y} P_{t_{1}}$ : for all measurable $A \subset E$,

$$
\bar{\mu}_{x, y}(A):=\inf _{A_{1} \cup A_{2}=A}\left(\delta_{x} P_{t_{1}} \mathbb{1}_{A_{1}}+\delta_{y} P_{t_{1}} \mathbb{1}_{A_{2}}\right) .
$$

The continuity of $(x, y) \mapsto \bar{\mu}_{x, y}(f)$ on $E^{2}$ holds as above.

Fix $x_{1} \in E$ and $d_{1}>0$ such that $\inf _{x \in B\left(x_{1}, d_{1}\right)} f(x)>0$. Then (3.3) entails

$$
\bar{\mu}_{x_{1}, x_{1}}(f)=\delta_{x_{1}} P_{t_{1}} f \geq \mathbb{P}_{x_{1}}\left(X_{t_{1}} \in B\left(x_{1}, d_{1}\right)\right) \inf _{x \in B\left(x_{1}, d_{1}\right)} f(x)>0 .
$$

Therefore, there exist $r_{1}, a_{1}>0$ such that $\bar{\mu}_{x, y}(f) \geq a_{1}$ for all $x, y \in$ $B\left(x_{1}, r_{1}\right)$.

Hence, for all nonnegative measurable $g: E \rightarrow \mathbb{R}_{+}$and for all $x, y \in E$ and all $u^{\prime} \in E$,

$$
\begin{aligned}
\delta_{x} P_{2 t_{1}} g & \geq \int_{E} \mathbb{1}_{u \in B\left(x_{1}, r_{1}\right)} P_{t_{1}} g(u) \delta_{x} P_{t_{1}}(d u) \\
& \geq \int_{E} \mathbb{1}_{u, u^{\prime} \in B\left(x_{1}, r_{1}\right)} \bar{\mu}_{u, u^{\prime}}(g) \delta_{x} P_{t_{1}}(d u) .
\end{aligned}
$$

Integrating both sides of the inequality w.r.t. $\delta_{y} P_{t_{1}}\left(d u^{\prime}\right)$, we obtain

$\delta_{x} P_{2 t_{1}} g \geq \delta_{x} P_{2 t_{1}} g \delta_{y} P_{t_{1}}(E) \geq \iint_{E \times E} \mathbb{1}_{u, u^{\prime} \in B\left(x_{1}, r_{1}\right)} \bar{\mu}_{u, u^{\prime}}(g) \delta_{x} P_{t_{1}}(d u) \delta_{y} P_{t_{1}}\left(d u^{\prime}\right)$.

Since this holds for all nonnegative measurable $g$ and since $\mu_{x, y}$ is the infimum measure between $\delta_{x} P_{2 t_{1}}$ and $\delta_{y} P_{2 t_{1}}$, by symmetry, we have proved that

$$
\mu_{x, y}(\cdot) \geq \iint_{E \times E} \mathbb{1}_{u, u^{\prime} \in B\left(x_{1}, r_{1}\right)} \bar{\mu}_{u, u^{\prime}}(\cdot) \delta_{x} P_{t_{1}}(d u) \delta_{y} P_{t_{1}}\left(d u^{\prime}\right)
$$


Therefore, (3.3) entails

$$
\mu_{x, y}(f) \geq a_{1} \mathbb{P}_{x}\left(X_{t_{1}} \in B\left(x_{1}, r_{1}\right)\right) \mathbb{P}_{y}\left(X_{t_{1}} \in B\left(x_{1}, r_{1}\right)\right)>0 .
$$

We now construct the measure $\nu_{x, y}$ of Condition (A1'). Using a similar computation as in (3.13) and integrating with respect to $\delta_{y} P_{2 t_{1}}\left(d u^{\prime}\right) / \delta_{y} P_{2 t_{1}} \mathbb{1}_{E}$, we obtain for all $x, y \in E$ and all nonnegative measurable $f: E \rightarrow \mathbb{R}_{+}$

$$
\delta_{x} P_{4 t_{1}} f \geq \iint_{K \times K} \mu_{u, u^{\prime}}(f) \delta_{x} P_{2 t_{1}}(d u) \frac{\delta_{y} P_{2 t_{1}}\left(d u^{\prime}\right)}{\delta_{y} P_{2 t_{1}} \mathbb{1}_{E}} .
$$

Since $\delta_{x} P_{4 t_{1}} \mathbb{1}_{E} \leq \delta_{x} P_{2 t_{1}} \mathbb{1}_{E}$,

$$
\begin{aligned}
\frac{\delta_{x} P_{4 t_{1}} f}{\delta_{x} P_{4 t_{1}} \mathbb{1}_{E}} & \geq \iint_{K \times K} \mu_{u, u^{\prime}}(f) \frac{\delta_{x} P_{2 t_{1}}(d u)}{\delta_{x} P_{2 t_{1}} \mathbb{1}_{E}} \frac{\delta_{y} P_{2 t_{1}}\left(d u^{\prime}\right)}{\delta_{y} P_{2 t_{1}} \mathbb{1}_{E}} \\
& =m_{x, y} \nu_{x, y}(f)
\end{aligned}
$$

where

$$
m_{x, y}:=\iint_{K \times K} \mu_{u, u^{\prime}}(E) \frac{\delta_{x} P_{2 t_{1}}(d u)}{\delta_{x} P_{2 t_{1}} \mathbb{1}_{E}} \frac{\delta_{y} P_{2 t_{1}}\left(d u^{\prime}\right)}{\delta_{y} P_{2 t_{1}} \mathbb{1}_{E}}
$$

and

$$
\nu_{x, y}:=\frac{1}{m_{x, y}} \iint_{K \times K} \mu_{u, u^{\prime}}(\cdot) \frac{\delta_{x} P_{2 t_{1}}(d u)}{\delta_{x} P_{2 t_{1}} \mathbb{1}_{E}} \frac{\delta_{y} P_{2 t_{1}}\left(d u^{\prime}\right)}{\delta_{y} P_{2 t_{1}} \mathbb{1}_{E}} .
$$

Note that

$$
\begin{aligned}
m_{x, y} & \geq \inf _{u, u^{\prime} \in K^{2}} \mu_{u, u^{\prime}}(E) \iint_{K \times K} \frac{\delta_{x} P_{2 t_{1}}(d u)}{\delta_{x} P_{2 t_{1}} \mathbb{1}_{E}} \frac{\delta_{y} P_{2 t_{1}}\left(d u^{\prime}\right)}{\delta_{y} P_{2 t_{1}} \mathbb{1}_{E}} \\
& \geq A^{2} \inf _{u, u^{\prime} \in K^{2}} \mu_{u, u^{\prime}}(E)>0,
\end{aligned}
$$

because of (3.10) and Lemma 3.3. Hence the probability measure $\nu_{x, y}$ is welldefined and we have proved (A1') for $t_{0}=4 t_{1}$ and $c_{1}=A^{2} \inf _{u, u^{\prime} \in K^{2}} \mu_{u, u^{\prime}}(E)$.

\subsubsection{Proof of (A2')}

Our goal is now to prove Condition (A2'). We first prove the following gradient estimate for $f=\mathbb{1}_{E}$.

Lemma 3.4. There exists a constant $C^{\prime \prime}>0$ such that, for all $t \geq 4 t_{1}$,

$$
\left\|\nabla P_{t} \mathbb{1}_{E}\right\|_{\infty} \leq C^{\prime \prime}\left\|P_{t} \mathbb{1}_{E}\right\|_{\infty}
$$


Note that, compared to (3.1), the difficulty is that we replace $\left\|\mathbb{1}_{E}\right\|_{\infty}$ by the smaller $\left\|P_{t_{1}} \mathbb{1}_{E}\right\|_{\infty}$ and that we extend this inequality to any time $t$ large enough.

Proof. We first use (3.10) to compute

$$
P_{4 t_{1}} \mathbb{1}_{E}(x) \geq \mathbb{P}_{x}\left(X_{2 t_{1}} \in K\right) \inf _{y \in K} \mathbb{P}_{y}\left(2 t_{1}<\tau_{\partial}\right) \geq m A \mathbb{P}_{x}\left(2 t_{1}<\tau_{\partial}\right),
$$

where $m:=\inf _{y \in K} \mathbb{P}_{y}\left(2 t_{1}<\tau_{\partial}\right)$ is positive because of Lemma 3.3. Integrating the last inequality with respect to $\left(\delta_{y} P_{t-4 t_{1}}\right)(d x)$ for any fixed $y \in E$ and $t \geq 4 t_{1}$, we deduce that

$$
\left\|P_{t} \mathbb{1}_{E}\right\|_{\infty} \geq m A\left\|P_{t-2 t_{1}} \mathbb{1}_{E}\right\|_{\infty} .
$$

Hence it follows from (3.6) that, for all $t \geq 4 t_{1}$,

$$
\begin{aligned}
\left\|\nabla P_{t} \mathbb{1}_{E}\right\|_{\infty} & =\left\|\nabla P_{t_{1}}\left(P_{t-t_{1}} \mathbb{1}_{E}\right)\right\|_{\infty} \leq C\left\|P_{t-t_{1}} \mathbb{1}_{E}\right\|_{\infty} \\
& \leq C\left\|P_{t-2 t_{1}} \mathbb{1}_{E}\right\|_{\infty} \leq \frac{C}{m A}\left\|P_{t} \mathbb{1}_{E}\right\|_{\infty} .
\end{aligned}
$$

This concludes the proof of Lemma 3.4.

This lemma implies that the function

$$
h_{t}: x \in E \cup\{\partial\} \mapsto \frac{P_{t} \mathbb{1}_{E}(x)}{\left\|P_{t} \mathbb{1}_{E}\right\|_{\infty}}
$$

is $C^{\prime \prime}$-Lipschitz for all $t \geq 4 t_{1}$. Since this function vanishes on $\partial$ and its maximum over $E$ is 1 , we deduce that, for any $t \geq 4 t_{1}$, there exists at least one point $z_{t} \in E$ such that $h_{t}\left(z_{t}\right)=1$. Since $h_{t}$ is $C^{\prime \prime}$-Lipschitz, we also deduce that $\rho\left(z_{t}, \partial\right) \geq 1 / C^{\prime \prime}$. Moreover, for all $x \in E$,

$$
\frac{P_{t} \mathbb{1}_{E}(x)}{\left\|P_{t} \mathbb{1}_{E}\right\|_{\infty}} \geq f_{z t}(x)
$$

where, for all $z \in E$ and $x \in E, f_{z}(x)=\left(1-C^{\prime \prime} \rho(x, z)\right) \vee 0$. We define the compact set $K^{\prime}=\left\{x \in E: \rho(x, \partial) \geq 1 / C^{\prime \prime}\right\}$ so that $z_{t} \in K^{\prime}$ for all $t \geq 4 t_{1}$. Then, for all $x, y \in E$ and for all $t \geq 4 t_{1}$, using the definition (3.14) of $\nu_{x, y}$,

$$
\begin{aligned}
\mathbb{P}_{\nu_{x, y}}\left(t<\tau_{\partial}\right) & \geq\left\|P_{t} \mathbb{1}_{E}\right\|_{\infty} \nu_{x, y}\left(f_{z_{t}}\right) \\
& =\frac{\left\|P_{t} \mathbb{1}_{E}\right\|_{\infty}}{m_{x, y}} \iint_{K \times K} \mu_{z, z^{\prime}}\left(f_{z t}\right) \frac{\delta_{x} P_{2 t_{1}}(d z)}{\delta_{x} P_{2 t_{1}} \mathbb{1}_{E}} \frac{\delta_{y} P_{2 t_{1}}\left(d z^{\prime}\right)}{\delta_{y} P_{2 t_{1}} \mathbb{1}_{E}}
\end{aligned}
$$


Since $z \mapsto f_{z}$ is Lipschitz for the $\|\cdot\|_{\infty}$ norm (indeed, $\left|f_{z}(x)-f_{z^{\prime}}(x)\right| \leq$ $C^{\prime \prime}\left|\rho(x, z)-\rho\left(x, z^{\prime}\right)\right| \leq C^{\prime \prime} \rho\left(z, z^{\prime}\right)$ for all $\left.x, z, z^{\prime} \in E^{3}\right)$, it follows from Lemma 3.3 that $(x, y, z) \mapsto \mu_{x, y}\left(f_{z}\right)$ is positive and continuous on $E^{3}$. Hence $c:=\inf _{x \in K, y \in K, z \in K^{\prime}} \mu_{x, y}\left(f_{z}\right)>0$ and, using that $m_{x, y} \leq 1$,

$$
\mathbb{P}_{\nu_{x, y}}\left(t<\tau_{\partial}\right) \geq c\left\|P_{t} \mathbb{1}_{E}\right\|_{\infty} \iint_{K \times K} \frac{\delta_{x} P_{2 t_{1}}(d z)}{\delta_{x} P_{2 t_{1}} \mathbb{1}_{E}} \frac{\delta_{y} P_{2 t_{1}}\left(d z^{\prime}\right)}{\delta_{y} P_{2 t_{1}} \mathbb{1}_{E}} \geq c A^{2}\left\|P_{t} \mathbb{1}_{E}\right\|_{\infty},
$$

where the last inequality follows from (3.10).

This entails Condition (A2') for all $t \geq 4 t_{1}$. For $t \leq 4 t_{1}$,

$$
\begin{aligned}
\mathbb{P}_{\nu_{x, y}}\left(t<\tau_{\partial}\right) & \geq \mathbb{P}_{\nu_{x, y}}\left(4 t_{1}<\tau_{\partial}\right) \geq c A^{2}\left\|P_{4 t_{1}} \mathbb{1}_{E}\right\|_{\infty} \\
& \geq c A^{2}\left\|P_{4 t_{1}} \mathbb{1}_{E}\right\|_{\infty} \sup _{z \in E} \mathbb{P}_{z}\left(t<\tau_{\partial}\right)>0 .
\end{aligned}
$$

This ends the proof of (A2') and hence of (1.1).

\subsubsection{Contraction in total variation norm}

It only remains to prove (3.4). By (1.2), we need to prove that there exists a constant $a>0$ such that, for all probability measure $\mu$ on $E$,

$$
c(\mu):=\inf _{t \geq 0} \frac{\mathbb{P}_{\mu}\left(t<\tau_{\partial}\right)}{\left\|P_{t} \mathbb{1}_{E}\right\|_{\infty}} \geq a \mu\left(\rho_{\partial}\right) .
$$

Because of the equivalence between (A) and (A') [6, Theorem 2.1], enlarging $t_{0}$ and reducing $c_{1}$ and $c_{2}$, one can assume without loss of generality that $\nu=\nu_{x, y}$ does not depend on $x, y \in E$. Then, using (A1) and (A2), we deduce that, for all $t \geq t_{0} \geq 4 t_{1}$,

$$
\begin{aligned}
\mathbb{P}_{\mu}\left(t<\tau_{\partial}\right) & =\mu\left(P_{t_{0}} P_{t-t_{0}} \mathbb{1}_{E}\right) \geq c_{1} \mathbb{P}_{\mu}\left(t_{0}<\tau_{\partial}\right) \mathbb{P}_{\nu}\left(t-t_{0}<\tau_{\partial}\right) \\
& \geq c_{1} c_{2}\left\|P_{t-t_{0}} \mathbb{1}_{E}\right\|_{\infty} \mathbb{P}_{\mu}\left(t_{0}<\tau_{\partial}\right) \geq c_{1} c_{2}\left\|P_{t} \mathbb{1}_{E}\right\|_{\infty} \mathbb{P}_{\mu}\left(t_{0}<\tau_{\partial}\right) .
\end{aligned}
$$

Now, using Assumption (3.2), we deduce that

$\mathbb{P}_{\mu}\left(t_{0}<\tau_{\partial}\right) \geq \mathbb{E}_{\mu}\left(\mathbb{1}_{T_{K}<t_{1}} \inf _{y \in K} \mathbb{P}_{y}\left(t_{0}<\tau_{\partial}\right)\right) \geq C^{\prime} \mu(\rho(\partial, \cdot)) \inf _{y \in K} \mathbb{P}_{y}\left(t_{0}<\tau_{\partial}\right)$,

where the constant $C^{\prime \prime}:=C^{\prime} \inf _{y \in K} \mathbb{P}_{y}\left(t_{0}<\tau_{\partial}\right)$ is positive. For $t \leq t_{0}$, the last inequality entails

$$
\mathbb{P}_{\mu}\left(t<\tau_{\partial}\right) \geq \mathbb{P}_{\mu}\left(t_{0}<\tau_{\partial}\right) \geq C^{\prime \prime} \mu(\rho(\partial, \cdot)) \geq C^{\prime \prime} \mu(\rho(\partial, \cdot))\left\|P_{t} \mathbb{1}_{E}\right\|_{\infty} .
$$

Hence (3.19) holds true with $a=c_{1} c_{2} C^{\prime \prime}$. This ends the proof of Theorem 3.1. 


\section{A Proof of $(1.2)$}

Let us assume that Condition (A) is satisfied. For all $t \geq 0$ and all probability measure $\pi$ on $E$, let $c_{t}(\pi):=\frac{\pi\left(P_{t} \mathbb{1}_{E}\right)}{\left\|P_{t} \mathbb{1}_{E}\right\|_{\infty}}$. In the proof of [6, Corollary 2.2], it is proved that, for all probability measures $\pi_{1}, \pi_{2}$ on $E$

$\left\|\mathbb{P}_{\pi_{1}}\left(X_{t} \in \cdot \mid t<\tau_{\partial}\right)-\mathbb{P}_{\pi_{2}}\left(X_{t} \in \cdot \mid t<\tau_{\partial}\right)\right\|_{T V} \leq \frac{\left(1-c_{1} c_{2}\right)^{\left\lfloor t / t_{0}\right\rfloor}}{c_{t}\left(\pi_{1}\right) \vee c_{t}\left(\pi_{2}\right)}\left\|\pi_{1}-\pi_{2}\right\|_{T V}$.

But

$$
\inf _{t \geq 0} c_{t}\left(\pi_{1}\right) \vee c_{t}\left(\pi_{2}\right) \geq\left(\inf _{t \geq 0} c_{t}\left(\pi_{1}\right)\right) \vee\left(\inf _{t \geq 0} c_{t}\left(\pi_{2}\right)\right)=c\left(\pi_{1}\right) \vee c\left(\pi_{2}\right)
$$

This ends the proof of (1.2).

\section{References}

[1] D. G. Aronson. Non-negative solutions of linear parabolic equations. Ann. Scuola Norm. Sup. Pisa (3), 22:607-694, 1968.

[2] K. Bogdan, T. Grzywny, and M. Ryznar. Heat kernel estimates for the fractional Laplacian with Dirichlet conditions. Ann. Probab., 38(5):1901-1923, 2010.

[3] P. Cattiaux, P. Collet, A. Lambert, S. Martínez, S. Méléard, and J. San Martín. Quasi-stationary distributions and diffusion models in population dynamics. Ann. Probab., 37(5):1926-1969, 2009.

[4] P. Cattiaux and S. Méléard. Competitive or weak cooperative stochastic lotka-volterra systems conditioned to non-extinction. J. Math. Biology, 60(6):797-829, 2010.

[5] N. Champagnat and D. Villemonais. Uniform convergence of conditional distributions for absorbed one-dimensional diffusions. ArXiv eprints, June 2015.

[6] N. Champagnat and D. Villemonais. Exponential convergence to quasistationary distribution and $Q$-process. Probab. Theory Related Fields, 164(1-2):243-283, 2016.

[7] N. Champagnat and D. Villemonais. Uniform convergence to the Qprocess. To appear in Electron. Commun. Prob., 2017. 
[8] Z.-Q. Chen, P. Kim, and R. Song. Heat kernel estimates for $\Delta+\Delta^{\alpha / 2}$ in $C^{1,1}$ open sets. J. Lond. Math. Soc. (2), 84(1):58-80, 2011.

[9] Z.-Q. Chen, P. Kim, and R. Song. Dirichlet heat kernel estimates for fractional Laplacian with gradient perturbation. Ann. Probab., 40(6):2483-2538, 2012.

[10] Z.-Q. Chen, P. Kim, and R. Song. Stability of Dirichlet heat kernel estimates for non-local operators under Feynman-Kac perturbation. Trans. Amer. Math. Soc., 367(7):5237-5270, 2015.

[11] E. B. Davies and B. Simon. Ultracontractivity and the heat kernel for Schrödinger operators and Dirichlet Laplacians. J. Funct. Anal., 59(2):335-395, 1984.

[12] P. Del Moral and D. Villemonais. Exponential mixing properties for time inhomogeneous diffusion processes with killing. Bernoulli Journal, 2016. To appear.

[13] E. B. Dynkin. Diffusions, superdiffusions and partial differential equations, volume 50 of American Mathematical Society Colloquium Publications. American Mathematical Society, Providence, RI, 2002.

[14] A. Hening and M. Kolb. Quasistationary distributions for onedimensional diffusions with singular boundary points. ArXiv e-prints, Sept. 2014.

[15] O. Kallenberg. Foundations of modern probability. Probability and its Applications (New York). Springer-Verlag, New York, second edition, 2002.

[16] K.-Y. Kim and P. Kim. Two-sided estimates for the transition densities of symmetric Markov processes dominated by stable-like processes in $C^{1, \eta}$ open sets. Stochastic Process. Appl., 124(9):3055-3083, 2014.

[17] P. Kim and R. Song. Estimates on Green functions and Schrödingertype equations for non-symmetric diffusions with measure-valued drifts. J. Math. Anal. Appl., 332(1):57-80, 2007.

[18] R. Knobloch and L. Partzsch. Uniform conditional ergodicity and intrinsic ultracontractivity. Potential Analysis, 33:107-136, 2010.

[19] M. Kolb and A. Wübker. Spectral analysis of diffusions with jump boundary. J. Funct. Anal., 261(7):1992-2012, 2011. 
[20] J.-F. Le Gall. Brownian motion, martingales, and stochastic calculus, volume 274 of Graduate Texts in Mathematics. Springer, french edition, 2016.

[21] J. Lierl and L. Saloff-Coste. The Dirichlet heat kernel in inner uniform domains: local results, compact domains and non-symmetric forms. $J$. Funct. Anal., 266(7):4189-4235, 2014.

[22] T. Lindvall and L. C. G. Rogers. Coupling of multidimensional diffusions by reflection. Ann. Probab., 14(3):860-872, 1986.

[23] J. Littin C. Uniqueness of quasistationary distributions and discrete spectra when $\infty$ is an entrance boundary and 0 is singular. J. Appl. Probab., 49(3):719-730, 2012.

[24] Y. Miura. Ultracontractivity for Markov semigroups and quasistationary distributions. Stoch. Anal. Appl., 32(4):591-601, 2014.

[25] D. Nualart. The Malliavin calculus and related topics. Probability and its Applications (New York). Springer-Verlag, Berlin, second edition, 2006.

[26] E. Priola and F.-Y. Wang. Gradient estimates for diffusion semigroups with singular coefficients. J. Funct. Anal., 236(1):244-264, 2006.

[27] D. W. Stroock and S. R. S. Varadhan. Multidimensional diffusion processes, volume 233 of Grundlehren der Mathematischen Wissenschaften [Fundamental Principles of Mathematical Sciences]. Springer-Verlag, Berlin, 1979.

[28] F.-Y. Wang. Sharp explicit lower bounds of heat kernels. Ann. Probab., 25(4):1995-2006, 1997.

[29] F.-Y. Wang. Gradient estimates of dirichlet heat semigroups and application to isoperimetric inequalities. Ann. Probab., 32(1A):424-440, 012004 .

[30] Q. S. Zhang. Gaussian bounds for the fundamental solutions of $\nabla(A \nabla u)+B \nabla u-u_{t}=0$. Manuscripta Math., 93(3):381-390, 1997.

[31] Q. S. Zhang. The boundary behavior of heat kernels of Dirichlet Laplacians. J. Differential Equations, 182(2):416-430, 2002. 